\title{
Application of He's Variational Iteration Method to Nonlinear Integro-Differential Equations
}

\author{
Ahmet Yildirim \\ Department of Mathematics, Science Faculty, Ege University, 35100 Bornova-İzmir, Turkey \\ Reprint requests to A. Y.; E-mail: ahmet.yildirim@ege.edu.tr \\ Z. Naturforsch. 65a, 418 - 422 (2010); received September 8, 2008 / February 6, 2009

\begin{abstract}
In this paper, an application of He's variational iteration method is applied to solve nonlinear solving various nonlinear integro-differential equations.
\end{abstract} \\ integro-differential equations. Some examples are given to illustrate the effectiveness of the method. \\ The results show that the method provides a straightforward and powerful mathematical tool for
}

Key words: He's Variational Iteration Method; Nonlinear Integro-Differential Equations.

\section{Introduction}

In recent years, some promising approximate analytical solutions are proposed, such as exp-function method [1], homotopy perturbation method [2-11], and variational iteration method (VIM) [12-17]. The variational iteration method is the most effective and convenient one for both weakly and strongly nonlinear equations. This method has been shown to effectively, easily, and accurately solve a large class of nonlinear problems with component converging rapidly to accurate solutions.

Avudainayagam and Vani [18] considered the application of wavelet bases in solving integro-differential equations. They introduced a new four-dimensional connection coefficient and an algorithm for its computation. They tested their algorithm by solving two simple pedagogic nonlinear integro-differential equations. El-Shaled [19] and Ghasemi et al. [2022] applied He's homotopy perturbation method to integro-differential equations. Ghasemi et al. [21, 22] and Kajani et al. [23] applied the Wavelet-Galerkin method and the sine-cosine wavelet method to integrodifferential equations. Also recently, Darania and Ebadian [24] applied the differential transform method to integro-differential equations.

In this paper, we propose VIM to solve the nonlinear integro-differential equations. The Volterra integrodifferential equation is given by

$$
u^{\prime}(x)=v(x)+\int_{0}^{x} k\left(x, t, u(t), u^{\prime}(t)\right) \mathrm{d} t
$$

and Fredholm type is given by

$$
u^{\prime}(x)=v(x)+\int_{a}^{b} k\left(x, t, u(t), u^{\prime}(t)\right) \mathrm{d} t .
$$

It was Wang and He [25] who first applied the variational iteration method to integro-differential equations. Lately Saberi-Nadjafi [26] found the method is a highly promising method for solving the system of integro-differential equations. Also $\mathrm{He}[27,28]$ gave new interpretations of the variational iteration method for solving integro-differential equations.

\section{He's Variational Iteration Method}

Now, to illustrate the basic concept of He's variational iteration method, we consider the following general nonlinear differential equation given in the form

$$
L u(t)+N u(t)=g(t)
$$

where $L$ is a linear operator, $N$ is a nonlinear operator, and $g(t)$ is a known analytical function. We can construct a correction functional according to the variational method as:

$$
u_{n+1}(t)=u_{n}(t)+\int_{0}^{t} \lambda\left(L u_{n}(\xi)+N \tilde{u}_{n}(\xi)-g(\xi)\right) \mathrm{d} \xi
$$

where $\lambda$ is a general Lagrange multiplier, which can be idendified optimally via the variational theory, the subscript $n$ denotes the $n$th approximation, and $\tilde{u}_{n}$ is considered as a restricted variation, namely $\delta \tilde{u}_{n}=0$ [12]. 
In the following examples, we will illustrate the usefulness and effectiveness of the proposed technique.

\section{Numerical Examples}

This section contains six examples of Volterra and Fredholm nonlinear integro-differential equations.

Example 1. Consider the following nonlinear integro-differential equation:

$$
u^{\prime}(x)=1+\int_{0}^{x} u(t) u^{\prime}(t) \mathrm{d} t
$$

for $x \in[0,1]$ with the exact solution

$$
u(x)=\sqrt{2} \tan \left(\frac{\sqrt{2}}{2} x\right) \text {. }
$$

Using He's variational iteration method, the correction functional can be written in the form

$u_{n+1}(x)=u_{n}(x)+\int_{0}^{x} \lambda(s)\left\{u_{n}^{\prime}(s)-1-\int_{0}^{s} u_{n}(p) \mathrm{d} p\right\} \mathrm{d} s$.

The stationary conditions

$$
1+\lambda=0, \quad \lambda^{\prime}=0
$$

follow immediately. This in turn gives

$$
\lambda=-1
$$

Substituting this value of the Lagrange multiplier, $\lambda=-1$, into the functional (6) gives the iteration formula

$$
\begin{aligned}
& u_{n+1}(x)= \\
& u_{n}(x)-\int_{0}^{x}\left\{u_{n}^{\prime}(s)-1-\int_{0}^{s} u_{n}(p) u_{n}^{\prime}(p) \mathrm{d} p\right\} \mathrm{d} s
\end{aligned}
$$

By VIM, let $L(u)=u^{\prime}(x)-v(x)=0$, we can choose $u_{0}(x)$ from the equation

$$
L(u)_{0}=u_{0}^{\prime}(x)-1=0 .
$$

We can select $u_{0}(x)=x$ from (10). Using this selection in (9), we obtain the following successive approximations:

$$
\begin{aligned}
& u_{0}(x)=x, \\
& u_{1}(x)=x+\frac{x^{3}}{6}, \\
& u_{2}(x)=x+\frac{x^{3}}{6}+\frac{x^{5}}{30}+\frac{x^{7}}{504},
\end{aligned}
$$

Table 1. Numerical results of Example 1.

\begin{tabular}{llll}
\hline$x$ & Exact Solution & VIM- $u_{3}$ & Absolute Error \\
\hline 0.0 & 0 & 0 & 0 \\
0.1 & 0.1001670006 & 0.1001670007 & $1 \times 10^{-9}$ \\
0.2 & 0.2013440870 & 0.2013440868 & $2 \times 10^{-9}$ \\
0.3 & 0.3045825026 & 0.3045824920 & $1.06 \times 10^{-8}$ \\
0.4 & 0.4110194227 & 0.4110192757 & $1.47 \times 10^{-7}$ \\
0.5 & 0.5219305152 & 0.5219293796 & $1.13 \times 10^{-6}$ \\
0.6 & 0.6387957040 & 0.6387895873 & $6.11 \times 10^{-6}$ \\
0.7 & 0.7633858019 & 0.7633600137 & $2.57 \times 10^{-5}$ \\
0.8 & 0.8978815369 & 0.8977903903 & $9.11 \times 10^{-5}$ \\
0.9 & 1.045043135 & 1.044760768 & $2.82 \times 10^{-4}$ \\
1.0 & 1.208460241 & 1.207669561 & $7.9 \times 10^{-4}$ \\
\hline
\end{tabular}

$$
\begin{aligned}
u_{3}(x)= & x+\frac{x^{3}}{6}+\frac{x^{5}}{30}+\frac{17 x^{7}}{2520}+\frac{19 x^{9}}{22680} \\
& +\frac{67 x^{11}}{831600}+\frac{x^{13}}{196560}+\frac{x^{15}}{7620480}
\end{aligned}
$$

The results and the corresponding absolute errors are presented in Table 1 (with third-order approximation (14)).

Table 1 shows that the numerical approximate solution has a high degree of accuracy. As we know, the more terms added to the approximate solution, the more accurate it will be. Although we only considered a third-order approximation, it achieves a high level of accuracy.

Example 2. Consider the following nonlinear integro-differential equation:

$$
u^{\prime}(x)=-\frac{1}{2}+\int_{0}^{x} u^{\prime 2}(t) \mathrm{d} t
$$

for $x \in[0,1]$ with the exact solution $u(x)=$ $-\ln \left(\frac{1}{2} x+1\right)$.

We can construct a variational iteration form for (15) in the form:

$u_{n+1}(x)=u_{n}(x)-\int_{0}^{x}\left\{u_{n}{ }^{\prime}(s)+\frac{1}{2}-\int_{0}^{s} u_{n}{ }^{2}(p) \mathrm{d} p\right\} \mathrm{d} s$.

By VIM, let $L(u)=u^{\prime}(x)-v(x)=0$, we can choose $u_{0}(x)$ from the equation

$$
L\left(u_{0}\right)=u_{0}{ }^{\prime}(x)+\frac{1}{2}=0 .
$$

We can select $u_{0}(x)=-\frac{x}{2}$ from (17). Using this selection in (16), we obtain the following successive approximations:

$$
u_{0}(x)=-\frac{x}{2}
$$


Table 2. Numerical results of Example 2.

\begin{tabular}{llll}
\hline$x$ & Exact Solution & VIM- $u_{3}$ & Absolute Error \\
\hline 0.0 & 0 & 0 & 0 \\
0.1 & -0.04879016417 & $-0,04879014498$ & $1.91 \times 10^{-8}$ \\
0.2 & -0.09531017980 & -0.09530961268 & $5.67 \times 10^{-7}$ \\
0.3 & -0.1397619424 & -0.1397579563 & $3.98 \times 10^{-6}$ \\
0.4 & -0.1823215568 & -0.1823059759 & $1.55 \times 10^{-5}$ \\
0.5 & -0.2231435513 & -0.2230993543 & $4.41 \times 10^{-5}$ \\
0.6 & -0.2623642645 & -0.2622618412 & $1.02 \times 10^{-4}$ \\
0.7 & -0.3001045925 & -0.2998980358 & $2.06 \times 10^{-4}$ \\
0.8 & -0.3364722366 & -0.3360958171 & $3.76 \times 10^{-4}$ \\
0.9 & -0.3715635564 & -0.3709284685 & $6.35 \times 10^{-4}$ \\
1.0 & -0.4054651081 & -0.4044565353 & $1 \times 10^{-3}$ \\
\hline
\end{tabular}

$$
\begin{aligned}
u_{1}(x)= & -\frac{x}{2}+\frac{x^{2}}{8} \\
u_{2}(x)= & -\frac{x}{2}+\frac{x^{2}}{8}-\frac{x^{3}}{24}+\frac{x^{4}}{192}, \\
u_{3}(x)= & -\frac{x}{2}+\frac{x^{2}}{8}-\frac{x^{3}}{24}+\frac{x^{4}}{64}+\frac{x^{5}}{240}+\frac{x^{6}}{1152} \\
& -\frac{x^{7}}{8064}+\frac{x^{8}}{129024},
\end{aligned}
$$

The results and the corresponding absolute errors are presented in Table 2 (with third-order approximation (21)).

Table 2 shows that the numerical approximate solution has a high degree of accuracy. As we know, the more terms added to the approximate solution, the more accurate it will be. Although we only considered a third-order approximation, it achieves a high level of accuracy.

Example 3. Consider the following second-order nonlinear integro-differential equation:

$$
u^{\prime \prime}(x)=\mathrm{e}^{x}-x+\int_{0}^{1} x t u(t) \mathrm{d} t
$$

with the initial conditions

$$
u(0)=1, \quad u^{\prime}(0)=1
$$

for $x \in[0,1]$ with the exact solution $u(x)=\mathrm{e}^{x}$.

Making $u_{n+1}(x)$ stationary with respect to $u_{n}(x)$, we can identify the Lagrange multiplier, which reads

$$
\lambda=s-x \text {. }
$$

So we can construct a variational iteration form for (22) in the form:

$$
\begin{aligned}
& u_{n+1}(x)=u_{n}(x) \\
& +\int_{0}^{x}(s-x)\left\{u_{n}^{\prime \prime}(s)-\mathrm{e}^{s}+s-\int_{0}^{1} s p u(p) \mathrm{d} p\right\} \mathrm{d} s .
\end{aligned}
$$

We begin with

$$
u_{0}(x)=\mathrm{e}^{x}(a+b x),
$$

where $a$ and $b$ are unknown constants to be further determined.

By the iteration formulation (25), we have

$$
\begin{aligned}
u_{1}(x)= & (a-1)+(a+b-1) x \\
& +\left(-\frac{1}{6}+\frac{1}{6} a-\frac{1}{3} b+\frac{1}{6} b e\right) x^{3}+\mathrm{e}^{x} .
\end{aligned}
$$

If the first-order approximate solution is enough, by the aid of the initial conditions (23), we can identify the unknown constants as

$$
a=1 \text { and } b=0 \text {. }
$$

So we obtain the following first-order approximate solution:

$$
u(x)=\mathrm{e}^{x}
$$

which is the exact solution of the problem.

Example 4. Now, we consider the following thirdorder nonlinear integro-differential equation:

$$
u^{\prime \prime \prime}(x)=\sin x-x-\int_{0}^{\pi / 2} x t u^{\prime}(t) \mathrm{d} t
$$

with the initial conditions

$$
u(0)=1, u^{\prime}(0)=0, \text { and } u^{\prime \prime}(0)=-1
$$

for $x \in[0, \pi / 2]$ with the exact solution $u(x)=\cos x$.

Making $u_{n+1}(x)$ stationary with respect to $u_{n}(x)$, we can identify the Lagrange multiplier, which reads

$$
\lambda=\frac{(s-x)^{2}}{2} .
$$

So we can construct a variational iteration form for (30) in the form:

$$
\begin{aligned}
u_{n+1}(x)=u_{n}(x)-\int_{0}^{x} & \frac{(s-x)^{2}}{2}\left\{u_{n}^{\prime \prime \prime}(s)-\sin s\right. \\
& \left.+s+\int_{0}^{\pi / 2} s p u^{\prime}(p) \mathrm{d} p\right\} \mathrm{d} s .
\end{aligned}
$$

We begin with

$$
u_{0}(x)=a+b x+c x^{2},
$$


where $a, b$ and $c$ are unknown constants to be further determined.

By the iteration formulation (33), we have

$$
u_{1}(x)=(a-1)+b x+\left(c+\frac{1}{2}\right) x^{2}+\cos x .
$$

If the first-order approximate solution is enough, by the aid of the initial conditions (31), we can identify the unknown constants as

$$
a=1, b=0, \text { and } c=-1 / 2 \text {. }
$$

So we obtain the following first-order approximate solution:

$$
u(x)=\cos x
$$

which is the exact solution of the problem.

Example 5. Finally, we consider the following fifthorder integro-differential equation:

$$
u^{(v)}(x)-u^{\prime}(x)=\int_{-1}^{1} u(t) \mathrm{d} t
$$

with initial conditions

$$
\begin{aligned}
& u(0)=0, u^{\prime}(0)=1, u^{\prime \prime}(0)=0, u^{\prime \prime \prime}(0)=-1, \\
& \text { and } u^{\prime v}(0)=0
\end{aligned}
$$

for $x \in[-1,1]$ with the exact solution $u(x)=\sin x$.

Making $u_{n+1}(x)$ stationary with respect to $u_{n}(x)$, we can identify the Lagrange multiplier, which reads

$$
\lambda=\frac{(s-x)^{4}}{24}
$$

So we can construct a variational iteration form for (38) in the form:

$$
\begin{aligned}
u_{n+1}(x)=u_{n}(x)-\int_{0}^{x} & \frac{(s-x)}{24}\left\{u_{n}{ }^{(v)}(s)\right. \\
& \left.-u_{n}{ }^{\prime}(s)-\int_{-1}^{1} u(p) \mathrm{d} p\right\} \mathrm{d} s .
\end{aligned}
$$

We begin with

$$
u_{0}(x)=a+b x+c x^{2}+d x^{3}+e x^{4},
$$

where $a, b, c, d$, and $e$ are unknown constants to be further determined.
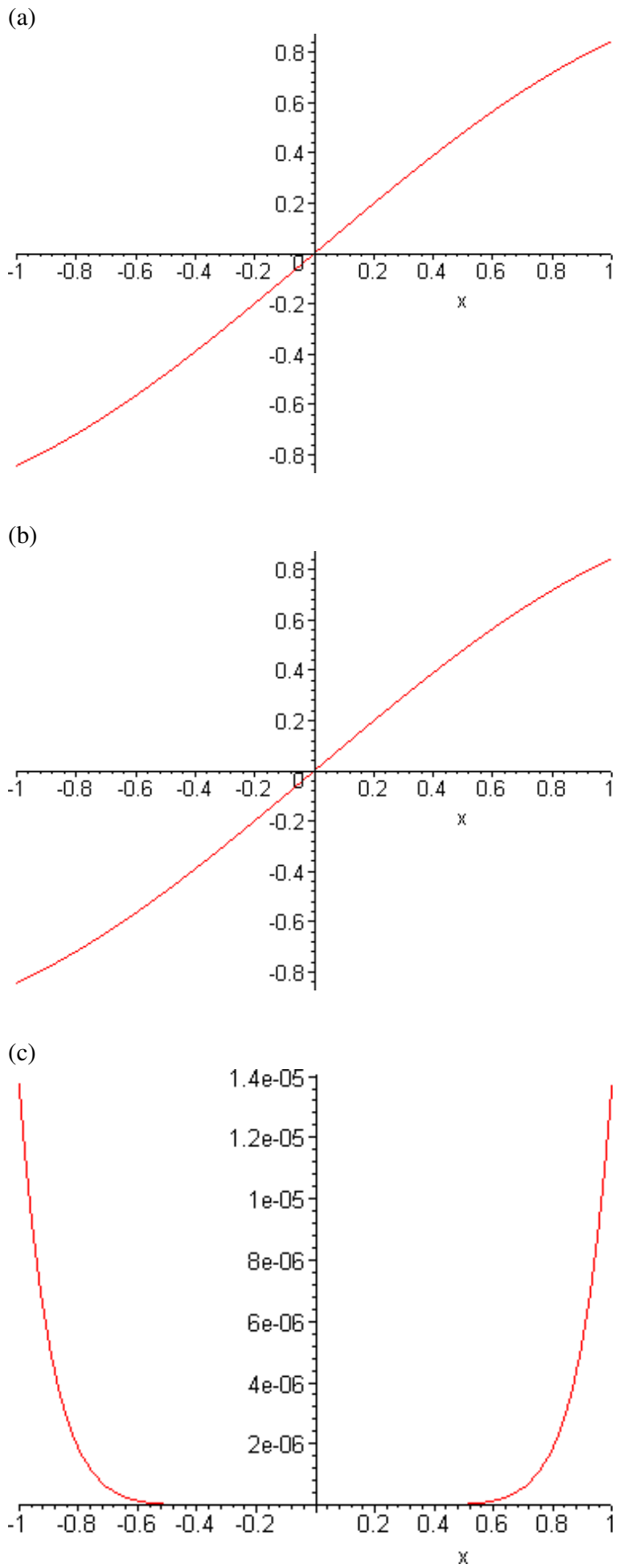

Fig. 1. (a) Exact Solution, (b) approximate Solution, (c) absolute error. 
By the iteration formulation (41), we have

$$
\begin{aligned}
u_{1}(x)= & a+b x+c x^{2}+d x^{3}+e x^{4} \\
& +\left(\frac{a}{60}+\frac{b}{120}+\frac{c}{180}\right) x^{5}+\left(\frac{a}{720}+\frac{c}{360}\right) x^{6} \\
& +\left(\frac{d}{280}+\frac{b}{2520}\right) x^{7}+\left(\frac{e}{240}+\frac{c}{6720}\right) x^{8} \\
& +\frac{d}{15120} x^{9}+\frac{e}{30240} x^{10}
\end{aligned}
$$

If the first-order approximate solution is enough, by the aid of the initial conditions (39), we can identify the unknown constants as

$$
a=0, b=1, c=0, d=-1 / 6, \text { and } e=0 .
$$

So we obtain the following first-order approximate solution:

$$
u_{1}(x)=x-\frac{x^{3}}{6}+\frac{x^{5}}{120}+\frac{x^{7}}{5040}+\frac{x^{9}}{90720} .
$$

The results and the corresponding absolute errors are presented in Figure 1 (with first-order approximation (45)). This figure shows that the numerical approx- imate solution has a high degree of accuracy. As we know, the more terms added to the approximate solution, the more accurate it will be. Although we only considered a first-order approximation, it achieves a high level of accuracy.

\section{Conclusion}

In this paper, we applied an application of He's variational iteration method for solving nonlinear integrodifferential equations. The method is extremely simple, easy to use and is very accurate for solving nonlinear integro-differential equation. The solution obtained by VIM is valid for not only weakly nonlinear equations, but also strong ones. Also, the method is a powerful tool to search for solutions of various linear/nonlinear problems. This variational iteration method will become a much more interesting method to solve nonlinear integro-differential equation in science and engineering.

\section{Acknowledgement}

The author sincerely thanks to unknown reviewers for their constructive comments and suggestions.
[1] J.H. He, X.H. Wu, Chaos, Solitons, and Fractals 30, 700 (2006).

[2] J. H. He, Appl. Math. Comput. 135, 73 (2003).

[3] J. H. He, Appl. Math. Comput. 151, 287 (2004).

[4] J. H. He, Chaos, Solitons, and Fractals 26, 695 (2005).

[5] J. H. He, Int. J. Nonlinear Sci. Numer. Simul. 6, 207 (2005).

[6] J. H. He, Phys. Lett. A 350, 87 (2006).

[7] T. Öziş and A.Yıldırım, Chaos, Solitons, and Fractals 34, 989 (2007).

[8] T. Öziş and A. Yıldırım, Int. J. Nonlinear Sci. Numer. Simul. 8, 243 (2007).

[9] T. Öziş and A. Yıldırım, Int. J. Nonlinear Sci. Numer. Simul. 8, 239 (2007).

[10] A. Yildırım, Int. J. Comput. Math., doi:10.1080/ 00207160802247646 (2008).

[11] A. Yıldırım and T. Öziş, Phys. Lett. A 369, 70 (2007).

[12] J. H. He, Int. J. Nonlinear Mech. 34, 699.

[13] J. H. He, Appl. Math. Comput. 114, 115 (2000).

[14] J.H. He and X.H. Wu, Chaos, Solitons, and Fractals 29, 108 (2006).

[15] T. Öziş and A. Yıldırım, J. Sound Vibr. 306, 372 (2007).
[16] A. Yıldırım, Math. Prob. Eng., Article ID 869614 (2008).

[17] A. Yıldırım, Comm. Nummer. Meth. Eng., doi:10. 1002/cnm.1154 (2008).

[18] A. Avudainayagam and C. Vani, Appl. Numer. Math. 32, 247 (2000).

[19] M. El-Shaled, Int. J. Nonlinear Sci. Numer. Simul. 6, 163 (2005).

[20] M. Ghasemi, M. T. Kajani, and E. Babolian, Appl. Math. Comput. 188, 538 (2007).

[21] M. Ghasemi, M. T. Kajani, and E. Babolian, Appl. Math. Comput. 188, 450 (2007).

[22] M. Ghasemi, M. T. Kajani, and E. Babolian, Comput. Math. Appl. 54, 1162 (2007).

[23] M. T. Kajani, M. Ghasemi, and E. Babolian, Appl. Math. Comput. 180, 569 (2006).

[24] P. Darania and A. Ebadian, Appl. Math. Comput. 188, 657 (2007).

[25] S. Q. Wang and J. H. He, Phys. Lett. A 367, 188 (2007).

[26] J. Saberi-Nadjafi and M. Tamamgar, Comput. Math. Appl. 56, 346 (2008).

[27] J.H. He and X. H. Wu, Comput. Math. Appl. 54, 881 (2007).

[28] J. H. He, J. Comput. Math. 207, 3 (2007). 


\title{
Fractional Diffusion Equation and External Forces: Solutions in a Confined Region
}

\author{
Ervin K. Lenzi ${ }^{\mathrm{a}}$, Roberto Rossato ${ }^{\mathrm{a}}$, Marcelo K. Lenzi ${ }^{\mathrm{b}}$, Luciano R. da Silva ${ }^{\mathrm{c}}$, \\ and Giane Gonçalves ${ }^{\mathrm{d}}$ \\ ${ }^{a}$ Departamento de Física, Universidade Estadual de Maringá, Av. Colombo 5790, \\ 87020-900, Maringá, Paraná, Brazil \\ b Departamento de Engenharia Química, Universidade Federal do Paraná, Setor de Tecnologia, \\ Curitiba, Paraná, Brazil \\ ${ }^{c}$ Departamento de Física, Universidade Federal do Rio Grande do Norte, 59072-970 Natal, \\ Rio Grande do Norte, Brazil \\ d Departamento de Engenharia Química, Universidade Estadual de Maringá, Av. Colombo 5790, \\ 87020-900, Maringá, Paraná, Brazil
}

Reprint requests to E. K. L.; E-mail: eklenzi@dfi.uem.br

Z. Naturforsch. 65a, 423 - 430 (2010); received March 20, 2009/ revised September 16, 2009

\begin{abstract}
We investigate the $\mathcal{N}$-dimensional fractional diffusion equation with radial symmetry by taking time dependent boundary conditions and external forces into account in a confined region. A spatial and time dependence on the diffusion coefficient is also considered. The results obtained show an anomalous dispersion of the solutions and non-usual behaviour for the survival probability.
\end{abstract}

Key words: Diffusion Equation; Confined Geometry; Anomalous Diffusion.

\section{Introduction}

Typical diffusive phenomena found in usual diffusion equation an appropriate description which has as main characteristic the square mean displacement with a linear dependence on time, i. e., $\left\langle(r-\langle r\rangle)^{2}\right\rangle \propto t$ [1]. This feature is deeply related to the central limit theorem and also to the Markovian nature of this stochastic process. However, a large number of experimental observations show that more complex processes, in which the mean-square displacement is not proportional to $t$, may also occur in nature. These situations can be found, for instance, in atom deposition into a porous substrate [2], diffusion of high molecular weight polyisopropylacrylamide in nanopores [3], highly confined hard disk fluid mixtures [4], fluctuating particle fluxes [5], conservative motion in a $d=2$ periodic potential [6], transport of fluid in porous media, diffusion on fractals [7], and many other interesting physical systems.

Several formalisms [8-11] have been employed to investigate these phenomena which present a nonconventional diffusion process. One of them is based on fractional diffusion equations [12-15], which have been widely applied to different physical scenarios. The advantage of these equations is the simple way to deal with boundary values problems and incorporate forced fields which in other approaches can lead to cumbersome situations. In addition, they may be related to continuous time random walk (CTRW) [16], Langevin equations [17], and master equations [18]. These features associated to fractional diffusion equations have motivated the study of their solutions in presence of, for instance, external forces [19-21], spatial dependent diffusion coefficients [22], reaction terms [23-26], cylindrical symmetry [27-29], and in confined regions [30-32]. Towards this direction, we address this work to the investigation of the $\mathcal{N}$ dimensional fractional diffusion equation with radial symmetry

$$
\begin{aligned}
\frac{\partial^{\gamma}}{\partial t^{\gamma}} \rho(r, t)= & \int_{0}^{t} \mathrm{~d} t^{\prime} \nabla \cdot\left(\mathcal{D}\left(r, t-t^{\prime}\right) \nabla \rho\left(r, t^{\prime}\right)\right) \\
& -\int_{0}^{t} \mathrm{~d}^{\prime} t \nabla \cdot\left(\bar{F}\left(r, t-t^{\prime}\right) \rho\left(r, t^{\prime}\right)\right),
\end{aligned}
$$

where $\mathcal{D}(r, t)$ represents the diffusion coefficient, $\bar{F}(r, t)$ is an external force acting on the system, and $0<\gamma \leq 1$ (subdiffusive case). The fractional time derivative representation considered here is the Caputo one [33] and the boundary conditions are $\rho(a, t)=$ $\phi_{a}(t)$ and $\rho(b, t)=\phi_{b}(t)$, where $\phi_{a}(t)$ and $\phi_{b}(t)$ are 
arbitrary time dependent functions. Consequently, the system described by (1) is restricted to the region $a \leq$ $r \leq b$ and subjected to time dependent boundary conditions.

In order to investigate the solutions of (1) subjected to time dependent boundary conditions, we use the Green function approach [34]. The solutions obtained within this formalism will be useful to investigate the dynamic properties related to the system and, in particular, clarify the role of the surface on the diffusive process. It is worth mentioning the importance of the solutions of (1) as they may be used to investigate, for example, adsorption-desorption processes with memory [35], situations characterized by reactive boundaries [36], and first time distribution in confined regions [37]. We start the analysis by considering (1) with $\mathcal{D}(r, t)=\overline{\mathcal{D}}(t) r^{-\theta}$, where $\overline{\mathcal{D}}(t)=t^{\eta-1} / \Gamma(\eta-1)$ with $\eta \geq 0$, in absence of external forces, i. e., $\bar{F}(r, t)=$ 0 . The solution obtained for this case as a particular case results found in [30-32] and may recover for $\alpha=1$ results found in [38] by taking the limit $a \rightarrow 0$ with $\rho(b, t)=0$. In this context, we also analyze the survival probability which for this case exhibits an anomalous behaviour. Afterwards, we incorporate the external force $\bar{F}(r, t)=-\left(k r-\mathcal{K} / r^{\varepsilon}\right) F^{\prime}(t) \hat{r}$, where $F^{\prime}(t)=t^{\eta-1} / \Gamma(\eta-1)$ and $\varepsilon=1+\theta$, in our analysis which is firstly addressed by considering $k=0$ and after this for the case $k \neq 0$. These developments are reported in Section 2, while conclusions are presented in Section 3.

\section{Fractional Diffusion Equation}

Let us consider (1) without external forces subjected to the time dependent boundary conditions $\rho(a, t)=$ $\phi_{a}(t)$ and $\rho(b, t)=\phi_{b}(t)$, with the initial condition given by an arbitrary function $\rho(r, 0)=\tilde{\rho}(r)$ which is initially normalized, i. e., $\int_{0}^{\infty} \mathrm{d} r r^{\mathcal{N}-1} \rho(r, t)=1$. By also considering a spatial dependence on the diffusion coefficient, i. e., it is given by $\mathcal{D}(r, t)=\overline{\mathcal{D}}(t) r^{-\theta}$, (1) may be written as

$$
\begin{aligned}
\frac{\partial^{\gamma}}{\partial t^{\gamma}} \rho(r, t)= & \frac{1}{r^{\mathcal{N}-1}} \\
& \cdot \int_{0}^{t} \mathrm{~d} t^{\prime} \frac{\partial}{\partial r}\left(\overline{\mathcal{D}}\left(t-t^{\prime}\right) r^{\mathcal{N}-1-\theta} \frac{\partial}{\partial r} \rho\left(r, t^{\prime}\right)\right)^{(2)}
\end{aligned}
$$

with $0<\gamma \leq 1$ and $\overline{\mathcal{D}}(t)=t^{\eta-1} / \Gamma(\eta-1)$. This equation extends the usual diffusion equation by incorporating fractional time derivatives and a spatial and time dependence on the diffusion coefficient. The spatial dependence adopted for the diffusion coefficient has been used to investigate several physical contexts such as diffusion on fractals [7], turbulence [39], and fast electrons in hot plasma in the presence of an electric field [40]. A direct consequence of the extensions incorporated in (2) accomplishing time dependent boundary conditions $\phi_{a}(t)$ and $\phi_{b}(t)$ is the anomalous spreading of the distribution and the quantities related to it. The solution anomalous behaviour is noticed by the behaviour of the mean-square displacement $\sigma^{2}=$ $\left\langle(r-\langle r\rangle)^{2}\right\rangle$ when compared to the usual one. In addition, it is interesting to note that for a suitable time dependence on the diffusion coefficient the solutions may exhibit different diffusive regimes and the choice of boundary conditions represents a key role regarding the presence of stationary solutions which, in particular, are equal to the usual one. This last feature shows that the fractional time derivative produces an anomalous relaxation of the solution to the stationary state, in contrast to the spatial fractional derivatives which lead to Lévy like distributions.

In order to obtain the solution for (2) and investigate the surface effect on the solution, i. e., how the boundary conditions may change the relaxation of the system, we use the Green function approach and the Laplace transform. Applying the Laplace transform to (2) and performing some calculations, it is possible to show that the solution is given by

$$
\begin{aligned}
& \rho(r, s)=-s^{\gamma-1} \int_{a}^{b} \mathrm{~d} r^{\prime} r^{\prime \mathcal{N}-1} G\left(r, r^{\prime} ; s\right) \bar{\rho}(r) \\
&+\overline{\mathcal{D}}(s)\left[\left.b^{\mathcal{N}-\theta-1} \phi_{b}(s) \frac{\partial}{\partial r^{\prime}} G\left(r, r^{\prime} ; s\right)\right|_{r^{\prime}=b}\right. \\
&\left.-\left.a^{\mathcal{N}-\theta-1} \phi_{a}(s) \frac{\partial}{\partial r^{\prime}} G\left(r, r^{\prime} ; s\right)\right|_{r^{\prime}=a}\right]
\end{aligned}
$$

where the last term represents the surface effect and determines the existence of an stationary solution. The Green function $G\left(r, r^{\prime} ; s\right)$ present in above equation is obtained by solving the equation

$$
\begin{aligned}
& \frac{1}{r^{\mathcal{N}-1}} \frac{\mathrm{d}}{\mathrm{d} r}\left(\overline{\mathcal{D}}(s) r^{\mathcal{N}-\theta-1} \frac{\mathrm{d}}{\mathrm{d} r} G\left(r, r^{\prime} ; s\right)\right) \\
& -s^{\gamma} G\left(r, r^{\prime} ; s\right)=\frac{1}{r^{\mathcal{N}-1}} \delta\left(r-r^{\prime}\right)
\end{aligned}
$$

subjected to the boundary conditions $\left.G\left(r, r^{\prime} ; s\right)\right|_{r=b}=0$ and $\left.G\left(r, r^{\prime} ; s\right)\right|_{r=a}=0$. Equation (4) may be solved by applying different procedures. We use the eigenfunctions of Sturm-Liouville related to the spatial operator 
of (4), i. e., the eigenfunctions which emerge from the equation $\partial_{r}\left(r^{\mathcal{N}-1-\theta} \partial_{r} \psi_{n}\left(k_{n}, r\right)\right)=-k_{n}^{2} r^{\mathcal{N}-1} \psi_{n}\left(k_{n}, r\right)$ with $\left.\psi_{n}\left(k_{n}, r\right)\right|_{r=a}=\left.\psi_{n}\left(k_{n}, r\right)\right|_{r=b}=0$. By employing this procedure it can be shown that the Green function for this case is given by

$$
\begin{aligned}
& G\left(r, r^{\prime} ; s\right)= \\
& \quad-\frac{1}{2+\theta} \sum_{n=1}^{\infty} \frac{\mathcal{N}_{n}}{s^{\gamma}+\overline{\mathcal{D}}(s) k_{n}^{2}} \psi_{n}\left(k_{n}, r^{\prime}\right) \psi_{n}\left(k_{n}, r\right)
\end{aligned}
$$

with

$$
\begin{aligned}
& \psi_{n}\left(k_{n}, r\right)=r^{\frac{1}{2}(2+\theta-\mathcal{N})} \\
& \cdot\left[\mathrm{J}_{|\alpha|}\left(\frac{2 k_{n}}{2+\theta} r^{\frac{1}{2}(2+\theta)}\right) \mathrm{N}_{|\alpha|}\left(\frac{2 k_{n}}{2+\theta} a^{\frac{1}{2}(2+\theta)}\right)\right. \\
& \left.\quad-\mathrm{J}_{|\alpha|}\left(\frac{2 k_{n}}{2+\theta} a^{\frac{1}{2}(2+\theta)}\right) \mathrm{N}_{|\alpha|}\left(\frac{2 k_{n}}{2+\theta} r^{\frac{1}{2}(2+\theta)}\right)\right],
\end{aligned}
$$

where $\alpha=\mathcal{N} /(2+\theta)-1, \mathrm{~J}_{\alpha}(x)$ and $\mathrm{N}_{\alpha}(x)$ are Bessel functions of first and second species. The eigenvalues $k_{n}$ are determined by using the equation

$$
\begin{aligned}
& \mathbf{J}_{|\alpha|}\left(\frac{2 k_{n}}{2+\theta} b^{\frac{1}{2}(2+\theta)}\right) \mathbf{N}_{|\alpha|}\left(\frac{2 k_{n}}{2+\theta} a^{\frac{1}{2}(2+\theta)}\right) \\
& -\mathbf{J}_{|\alpha|}\left(\frac{2 k_{n}}{2+\theta} a^{\frac{1}{2}(2+\theta)}\right) \mathrm{N}_{|\alpha|}\left(\frac{2 k_{n}}{2+\theta} b^{\frac{1}{2}(2+\theta)}\right)=0
\end{aligned}
$$

and

$$
\mathcal{N}_{n}=\frac{\pi^{2} k_{n}^{2}}{\mathrm{~J}_{|\alpha|}^{2}\left(\frac{2 k_{n}}{2+\theta} a^{\frac{1}{2}(2+\theta)}\right) / \mathrm{J}_{|\alpha|}^{2}\left(\frac{2 k_{n}}{2+\theta} b^{\frac{1}{2}(2+\theta)}\right)-1} .
$$

Applying the inverse Laplace transform (3) and (5) with $\overline{\mathcal{D}}(s)=\mathcal{D} / s^{\eta} \quad\left(\overline{\mathcal{D}}(t)=\mathcal{D} t^{\eta-1} / \Gamma(\eta-1)\right)$, we obtain that

$$
\begin{aligned}
& \rho(r, t)=-\frac{1}{\Gamma(1-\gamma)} \int_{0}^{t} \mathrm{~d} t^{\prime} \frac{1}{\left(t-t^{\prime}\right)^{\gamma-1}} \\
& \cdot \int_{a}^{b} \mathrm{~d} r^{\prime} r^{\prime \mathcal{N}-1} G\left(r, r^{\prime} ; s\right) \bar{\rho}(r)+\int_{0}^{t} \mathrm{~d} \overline{\mathcal{D}} \overline{\mathcal{D}}(t-\bar{t}) \\
& \cdot\left\{\left.b^{\mathcal{N}-\theta-1} \int_{0}^{\bar{t}} \mathrm{~d} t^{\prime} \phi_{b}\left(\bar{t}-t^{\prime}\right) \frac{\partial}{\partial r^{\prime}} G\left(r, r^{\prime} ; t^{\prime}\right)\right|_{r^{\prime}=b}\right. \\
& \left.-\left.a^{\mathcal{N}-\theta-1} \int_{0}^{\bar{t}} \mathrm{~d} t^{\prime} \phi_{a}\left(\bar{t}-t^{\prime}\right) \frac{\partial}{\partial r^{\prime}} G\left(r, r^{\prime} ; t^{\prime}\right)\right|_{r^{\prime}=a}\right\}
\end{aligned}
$$

with

$$
\begin{array}{r}
G\left(r, r^{\prime} ; t\right)=-\frac{t^{\gamma-1}}{2+\theta} \sum_{n=1}^{\infty} \mathcal{N}_{n} \psi_{n}\left(k_{n}, r^{\prime}\right) \psi_{n}\left(k_{n}, r\right) \\
\cdot \mathrm{E}_{\gamma+\eta, \gamma}\left(-D k_{n}^{2} t^{\gamma+\eta}\right),
\end{array}
$$

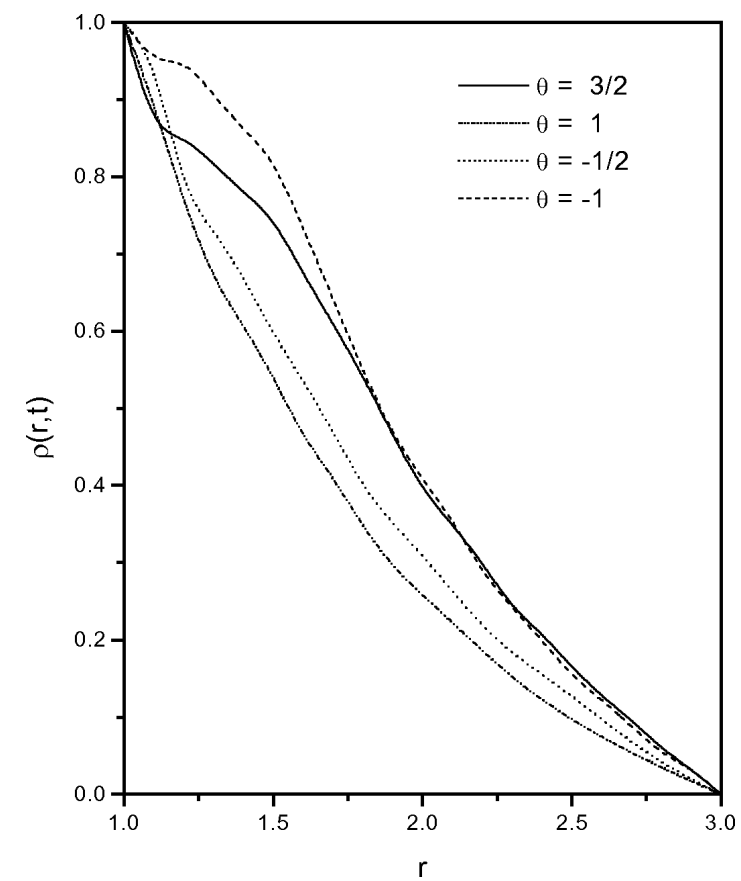

Fig. 1. Behaviour of $\rho(r, t)$ versus $r$ obtained from equation (9) for typical values of $\theta$, accomplishing $\Phi_{a}(t)=1$ and $\Phi_{b}(t)=0$. We consider, for simplicity, $\mathcal{D}=1, \mathcal{N}=2$, $a=1, b=3, t=1, \gamma+\eta=1 / 2$, and the initial condition $\rho(r, 0)=\delta(r-3 / 2) / r^{\mathcal{N}-1}$. Note that the value of $\theta$ modifies the diffusive process of the system and for $\theta<0$ the diffusion is faster than $\theta>0$.

where $\mathrm{E}_{\alpha, \beta}(x)=\sum_{n=0}^{\infty} x^{n} / \Gamma(\beta+\alpha n)$ is the generalized Mittag-Leffler function [33] (see Fig. 1). The presence of this function in above equation indicates an anomalous dispersion of the solution due to the presence of fractional time derivatives in (2). In particular, it is interesting to note that the time dependence in the diffusion coefficient also produces an anomalous dispersion of the solution and, in particular, depending on the choice of the time dependence different diffusive regimes may be obtained as mentioned before. The anomalous spreading may be verified by analyzing the dispersion relation, i. e., $\sigma^{2}=\left\langle(r-\langle r\rangle)^{2}\right\rangle$. For the case characterized by the boundary condition given by $\rho(a, t)=\rho(b, t)=0$, the initial condition $\rho(r, 0)=\delta(r-\tilde{r}) / t^{\mathcal{N}-1}$ and $\theta=0$, we have that

$$
\sigma^{2}(t)=\left\langle r^{2}\right\rangle-(2-\mathcal{S}(t))\langle r\rangle^{2}
$$

(see Fig. 2) with

$$
\begin{array}{r}
\left\langle r^{2}\right\rangle=\sum_{n=1}^{\infty} \frac{\mathcal{N}_{n}}{\pi k_{n}^{2}}\left(b^{\frac{\mathcal{N}}{2}+1} \frac{\mathrm{J}_{|\alpha|}\left(k_{n} a\right)}{\mathbf{J}_{|\alpha|}\left(k_{n} b\right)}-a^{\frac{\mathcal{N}}{2}+1}\right) \\
\cdot \psi_{n}\left(\tilde{r}, k_{n}\right) \mathrm{E}_{\gamma+\eta}\left(-D k_{n}^{2} \gamma^{\gamma+\eta}\right)
\end{array}
$$




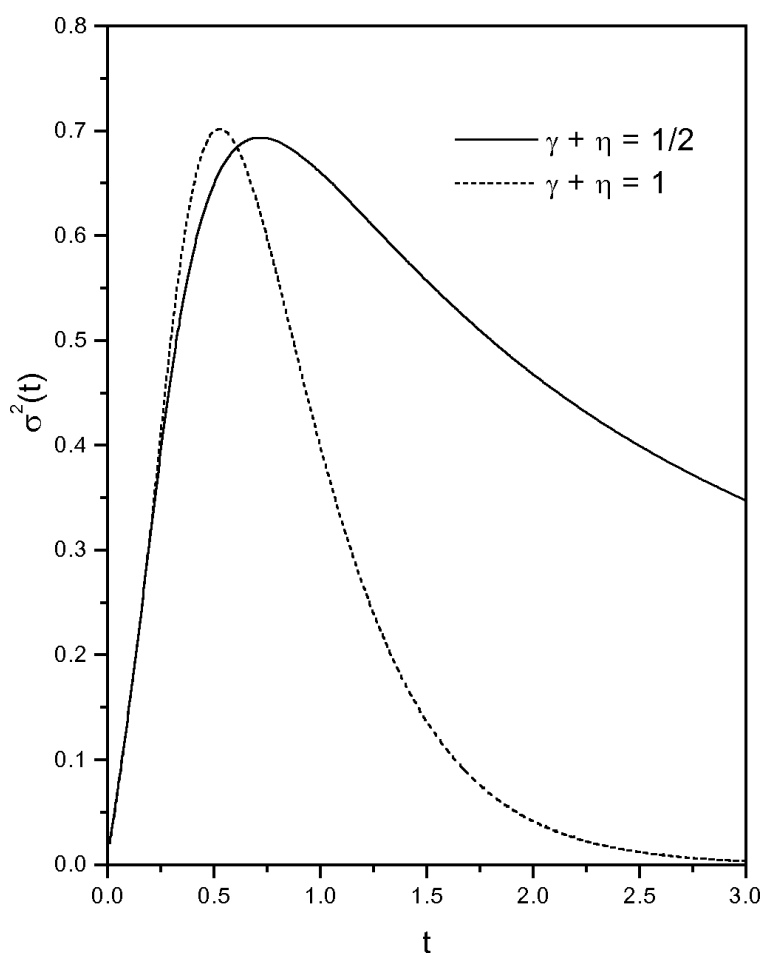

Fig. 2. Behaviour of $\sigma^{2}(t)$ versus $t$ in (11) for typical values of $\gamma+\eta$ by considering $\mathcal{D}=1, \mathcal{N}=1, a=1, b=3$, and $\rho(r, 0)=\delta(r-2) / r^{\mathcal{N}-1}$.

$$
\begin{gathered}
-\sum_{n=1}^{\infty} \frac{2 \mathcal{N} \mathcal{N}_{n}}{\pi k_{n}^{4}}\left(b^{\frac{\mathcal{N}}{2}-1} \frac{\mathbf{J}_{|\alpha|}\left(k_{n} a\right)}{\mathbf{J}_{|\alpha|}\left(k_{n} b\right)}-a^{\frac{\mathcal{N}}{2}-1}\right) \\
\cdot \psi_{n}\left(\tilde{r}, k_{n}\right) \mathrm{E}_{\gamma+\eta}\left(-D k_{n}^{2} t^{\gamma+\eta}\right), \\
\langle r\rangle=\sum_{n=1}^{\infty} \frac{\mathcal{N}_{n}}{\pi k_{n}^{2}}\left(b^{\frac{\mathcal{N}}{2}} \frac{\mathbf{J}_{|\alpha|}\left(k_{n} a\right)}{\mathbf{J}_{|\alpha|}\left(k_{n} b\right)}-a^{\frac{\mathcal{N}}{2}}\right) \\
-\psi_{n}\left(\tilde{r}, k_{n}\right) \mathrm{E}_{\gamma+\eta}\left(-D k_{n}^{2} t^{\gamma+\eta}\right) \\
-\sum_{n=1}^{\infty} \frac{\mathcal{N}_{n}}{\pi k_{n}^{4}}\left(b^{\frac{\mathcal{N}}{2}-1} \frac{\mathbf{J}_{|\alpha|}\left(k_{n} a\right)}{\mathbf{J}_{|\alpha|}\left(k_{n} b\right)}-a^{\frac{\mathcal{N}}{2}-1}\right) \\
\cdot \psi_{n}\left(\tilde{r}, k_{n}\right) \mathrm{E}_{\gamma+\eta}\left(-D k_{n}^{2} t^{\gamma+\eta}\right),
\end{gathered}
$$

and

$$
\begin{array}{r}
\mathcal{S}(t)=\sum_{n=1}^{\infty} \frac{\mathcal{N}_{n}}{\pi k_{n}^{2}}\left(b^{\frac{\mathcal{N}}{2}-1} \frac{\mathrm{J}_{|\alpha|}\left(k_{n} a\right)}{\mathbf{J}_{|\alpha|}\left(k_{n} b\right)}-a^{\frac{\mathcal{N}}{2}-1}\right) \\
\cdot \psi_{n}\left(\tilde{r}, k_{n}\right) \mathrm{E}_{\gamma+\eta}\left(-D k_{n}^{2} t^{\gamma+\eta}\right)
\end{array}
$$

(see Fig. 3). Note that the quantity $\mathcal{S}(t)=\int_{a}^{b} \mathrm{~d} r r^{\mathcal{N}-1}$ $\cdot \rho(r, t)$ is the survival probability [41], i. e., it is related to the quantity of particles which are present in

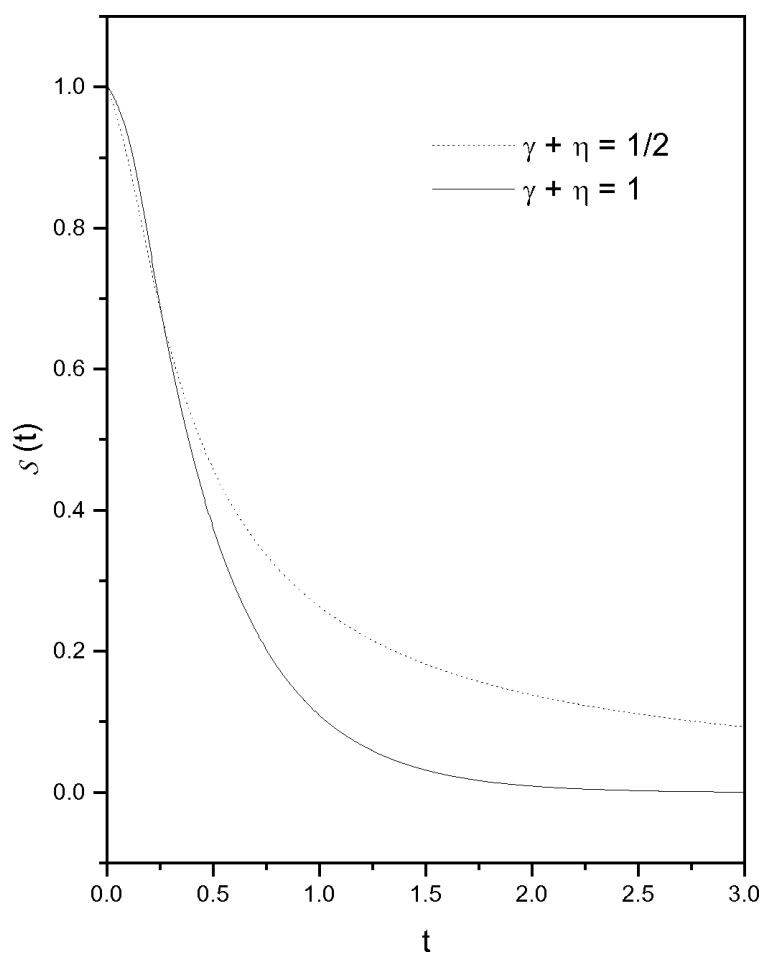

Fig. 3. Behaviour of $\mathcal{S}(t)$ versus $t$ in (14) for typical values of $\gamma+\eta$ by considering $\Phi_{a}(t)=1, \Phi_{b}(t)=0, \mathcal{D}=1$, $\mathcal{N}=1, a=1, b=3$, and the initial condition $\rho(r, 0)=$ $\delta(r-2) / r^{\mathcal{N}-1}$.

the region defined by the interval $a \leq r \leq b$. This manner, the quantity $1-\mathcal{S}(t)$ gives the quantity of particles absorbed by the surfaces, i. e., removed of the system.

Now, let us incorporate in our analysis the external force $F(r, t)=\mathcal{K} / r^{\varepsilon}(\varepsilon=1+\theta)$ which can be derived from a power law potential and has the logarithmic potential as particular case and a reaction term represented by $\tilde{\alpha}(r, t)$. In this way, we expect to extend the previous solution to a broader context in order to make possible the investigation of, for example, drug absorption or deliver [42], tumor development [43], and heat production [44]. Employing the previous procedure, the solution for (1) subject to the previous external force and with reaction term is given by

$$
\begin{aligned}
& \rho(r, t)= \\
& -\frac{1}{\Gamma(1-\gamma)} \int_{0}^{t} \mathrm{~d} t^{\prime} \frac{1}{\left(t-t^{\prime}\right)^{\gamma-1}} \int_{a}^{b} \mathrm{~d} r^{\prime \mathcal{N}-1} \widetilde{G}\left(r, r^{\prime} ; t^{\prime}\right) \bar{\rho}(r) \\
& -\int_{0}^{t} \mathrm{~d} t^{\prime} \int_{a}^{b} \mathrm{~d} r^{\prime \mathcal{N}-1} \widetilde{G}\left(r, r^{\prime} ; t-t^{\prime}\right) \tilde{\alpha}\left(r^{\prime}, t^{\prime}\right)
\end{aligned}
$$




$$
+\int_{0}^{t} \mathrm{~d} \bar{\tau} \overline{\mathcal{D}}(t-\bar{t})\left\{\left.b^{\mathcal{N}-\theta-1} \int_{0}^{\bar{t}} \mathrm{~d} t^{\prime} \phi_{b}\left(\bar{t}-t^{\prime}\right) \frac{\partial}{\partial r^{\prime}} \widetilde{G}\left(r, r^{\prime} ; t^{\prime}\right)\right|_{r=b}-\left.a^{\mathcal{N}-\theta-1} \int_{0}^{\bar{t}} \mathrm{~d} t^{\prime} \phi_{a}\left(\bar{t}-t^{\prime}\right) \frac{\partial}{\partial r^{\prime}} \widetilde{G}\left(r, r^{\prime} ; t^{\prime}\right)\right|_{r=a}\right\}
$$

with the Green function given by

$$
\widetilde{G}\left(r, r^{\prime} ; t\right)=-\frac{t^{\gamma-1} r^{\frac{\mathcal{K}}{\mathcal{D}}}}{2+\theta} \sum_{n=1}^{\infty} \widetilde{\mathcal{N}}_{n} \tilde{\psi}_{n}\left(k_{n}, r^{\prime}\right) \tilde{\psi}_{n}\left(k_{n}, r\right) \mathrm{E}_{\gamma+\eta, \gamma}\left(-D k_{n}^{2} t^{\gamma}\right),
$$

where

$$
\begin{aligned}
\tilde{\psi}_{n}\left(k_{n}, r\right)=r^{\frac{1}{2}\left(2+\theta-\mathcal{N}-\frac{\mathcal{K}}{\mathcal{D}}\right)[} & \mathbf{J}_{|v|}\left(\frac{2 k_{n}}{2+\theta} r^{\frac{1}{2}(2+\theta)}\right) \mathrm{N}_{|v|}\left(\frac{2 k_{n}}{2+\theta} a^{\frac{1}{2}(2+\theta)}\right) \\
& \left.-\mathbf{J}_{|v|}\left(\frac{2 k_{n}}{2+\theta} a^{\frac{1}{2}(2+\theta)}\right)-\mathrm{N}_{|v|}\left(\frac{2 k_{n}}{2+\theta} r^{\frac{1}{2}(2+\theta)}\right)\right]
\end{aligned}
$$

with $v=(\mathcal{N}+\mathcal{K} / \mathcal{D}) /(2+\theta)-1$. The $k_{n}$ are obtained from (7) by replacing the index $\alpha$ of the Bessel functions by the index $v$ and finally, with the normalization factor given by

$$
\widetilde{\mathcal{N}}_{n}=\frac{\pi^{2} k_{n}^{2}}{\mathrm{~J}_{|v|}^{2}\left(\frac{2 k_{n}}{2+\theta} a^{\frac{1}{2}(2+\theta)}\right) / \mathrm{J}_{|v|}^{2}\left(\frac{2 k_{n}}{2+\theta} b^{\frac{1}{2}(2+\theta)}\right)-1} .
$$

This result extends the results presented in [30-32] to the $\mathcal{N}$-dimensional case, and recovers the results obtained in [38] when the external force is absent for $\eta=0$. In addition, the results found in [29] and [45] are also a particular case of the above equations. By applying the limit $b \rightarrow \infty$ in (15) and also considering the boundary condition $\rho(\infty, t)=0$, we obtain an interesting result. It is given by

$$
\begin{aligned}
\rho(r, t)= & -\frac{1}{\Gamma(1-\gamma)} \int_{0}^{t} \mathrm{~d} \tilde{t} \frac{1}{(t-\tilde{t})^{\gamma}} \int_{0}^{\infty} \mathrm{d} \tilde{r} \tilde{r}^{\mathcal{N}-1} \tilde{\rho}(\tilde{r}) G(r, \tilde{r} ; s) \\
& -\int_{0}^{t} \mathrm{~d} t^{\prime} \int_{a}^{\infty} \mathrm{d} r^{\prime} r^{\prime \mathcal{N}-1} \widetilde{G}\left(r, r^{\prime} ; t-t^{\prime}\right) \alpha\left(r^{\prime}, t^{\prime}\right)-\left.a^{\mathcal{N}-\theta-1} \int_{0}^{t} \mathrm{~d} \bar{t} \int_{0}^{\bar{t}} \mathrm{~d} t^{\prime} \mathcal{D}(t-\bar{t}) \phi_{a}\left(\bar{t}-t^{\prime}\right) \frac{\partial}{\partial r^{\prime}} \widetilde{\mathcal{G}}\left(r, r^{\prime} ; t^{\prime}\right)\right|_{r^{\prime}=a}
\end{aligned}
$$

with the Green function

$$
\mathcal{G}(r, \tilde{r} ; t)=-\frac{2 t^{\gamma-1}}{2+\theta} \int_{0}^{\infty} \mathrm{d} k \frac{k \psi(\tilde{r}, k) \psi(r, k) \mathrm{E}_{\gamma+\eta, \gamma}\left(-k^{2} t^{\gamma+\eta}\right)}{\mathrm{J}_{|v|}^{2}\left(\frac{2 k}{2+\theta} a^{\frac{1}{2}(2+\theta)}\right)+\mathrm{N}_{|v|}^{2}\left(\frac{2 k}{2+\theta} a^{\frac{1}{2}(2+\theta)}\right)} .
$$

Note that this solution is restricted to the interval $a \leq r<\infty$ and may be extended in order to cover the interval $0 \leq r<\infty$. In this direction, we need to take the limit $a \rightarrow 0$ and the restriction $2+\theta-\mathcal{N}-\mathcal{K} / \mathcal{D}>0$ in $(20)$ to preserve the boundary conditions $\mathcal{G}(0, \tilde{r}, t)=0$ and $\mathcal{G}(\infty, \tilde{r}, t)=0$ for the Green function. These conditions lead to the Green function

$$
\mathcal{G}(r, \tilde{r} ; t)=-\frac{2 t^{\gamma-1}}{2+\theta}(r \tilde{r})^{\frac{1}{2}\left(2+\theta-\mathcal{N}-\frac{\mathcal{K}}{\mathcal{D}}\right)} \int_{0}^{\infty} \mathrm{d} k k \mathbf{J}_{|v|}\left(\frac{2 k}{2+\theta} r^{\frac{1}{2}(2+\theta)}\right) \mathbf{J}_{|v|}\left(\frac{2 k}{2+\theta} \tilde{r}^{\frac{1}{2}(2+\theta)}\right) \mathrm{E}_{\gamma+\eta, \gamma}\left(-k^{2} t^{\gamma}\right) .
$$

For the case $v=1 / 2$ and $\eta=0$ the above equation may be simplified to

$$
\begin{aligned}
\mathcal{G}(r, \tilde{r} ; t)= & -\frac{1}{2 t} \sqrt{\frac{t^{\gamma}}{\mathcal{D}}}(r \tilde{r})^{\frac{1}{2}\left(\frac{1}{2}(2+\theta)-\mathcal{N}-\frac{\mathcal{K}}{\mathcal{D}}\right)}\left\{\mathbf{H}_{11}^{10}\left[\frac{2}{(2+\theta) \sqrt{\mathcal{D} t} \gamma}\left|r^{\frac{1}{2}(2+\theta)}-\tilde{r}^{\frac{1}{2}(2+\theta) \mid}\right|_{(0,1)}^{(\gamma / 2, \gamma / 2)}\right]\right. \\
& \left.-\mathbf{H}_{11}^{10}\left[\left.\frac{2}{(2+\theta) \sqrt{\mathcal{D} t}}\left|r^{\frac{1}{2}(2+\theta)}+\tilde{r}^{\frac{1}{2}(2+\theta)}\right|\right|_{(0,1)} ^{(\gamma / 2, \gamma / 2)}\right]\right\}
\end{aligned}
$$

where $\mathbf{H}_{\mathrm{p}, \mathrm{q}}^{\mathrm{m}, \mathrm{n}}\left[\left.x\right|_{\left(b_{q}, B_{q}\right)} ^{\left(a_{p}, A_{p}\right)}\right]$ is the H Fox function [46]. For $\gamma=1$ with $\eta=0$ we have 


$$
\mathcal{G}(r, \tilde{r} ; t)=\frac{1}{(2+\theta) \mathcal{D} t}(r \tilde{r})^{\frac{1}{2}\left(2+\theta-\mathcal{N}-\frac{\mathcal{K}}{\mathcal{D}}\right)} \mathrm{e}^{-\frac{1}{(2+\theta)^{2} \mathcal{D} t}\left(r^{2+\theta}+\tilde{r}^{2+\theta}\right)} \mathrm{I}_{|v|}\left(\frac{(r \tilde{r})^{2+\theta}}{(2+\theta)^{2} \mathcal{D} t}\right)
$$

where $\mathrm{I}_{v}(x)$ is a Bessel function of modified argument (see Fig. 4).

The external force worked out above may be extended by incorporating a linear term, i. e., $-k r$, which results in $F(r, t)=-k r+\mathcal{K} / r^{\varepsilon}$ with $\varepsilon=1+\theta$. The solution for this case is formally given by (15) with the Green function

$$
\mathcal{G}(r, \bar{r}, t)=-r^{\mathcal{\mathcal { K }}} \mathrm{e}^{-\frac{k r^{2}+\theta}{(2+\theta) \mathcal{D}}} t^{\gamma-1} \sum_{n=1}^{\infty} \overline{\mathcal{N}}_{n} \bar{\psi}_{n}\left(r, \lambda_{n}\right) \bar{\psi}_{n}\left(\bar{r}, \lambda_{n}\right) \mathrm{E}_{\gamma+\eta, \gamma}\left(-\mathcal{D} \lambda_{n}^{2} t^{\gamma+\eta}\right)
$$

where

$$
\bar{\psi}_{n}\left(r, \lambda_{n}\right)=\Phi\left(\bar{\lambda}_{n}, \beta ; \frac{k r^{2+\theta}}{(2+\theta) \mathcal{D}}\right) \Psi\left(\bar{\lambda}_{n}, \beta ; \frac{k a^{2+\theta}}{(2+\theta) \mathcal{D}}\right)-\Phi\left(\bar{\lambda}_{n}, \beta ; \frac{k a^{2+\theta}}{(2+\theta) \mathcal{D}}\right) \Psi\left(\bar{\lambda}_{n}, \beta ; \frac{k r^{2+\theta}}{(2+\theta) \mathcal{D}}\right)
$$

with $\bar{\lambda}_{n}=-\lambda_{n} /(2+\theta) k, \quad \beta=(\mathcal{K}+\mathcal{N D}) /((2+\theta) \mathcal{D}) . \Phi(\alpha, \beta ; x)$ is the confluent hypergeometric function,

$$
\Psi(\alpha, \beta ; x)=\frac{\Gamma(1-\beta)}{\Gamma(1+\alpha-\beta)} \Phi(\alpha, \beta ; x)+\frac{\Gamma(\beta-1)}{\Gamma(\alpha)} x^{1-\beta} \Phi(1+\alpha-\beta, 2-\beta ; x) .
$$

The eigenvalues $\lambda_{n}$ are determined by the equation

$$
\Phi\left(\bar{\lambda}_{n}, \beta ; \frac{k b^{2+\theta}}{(2+\theta) \mathcal{D}}\right) \Psi\left(\bar{\lambda}_{n}, \beta ; \frac{k a^{2+\theta}}{(2+\theta) \mathcal{D}}\right)-\Phi\left(\bar{\lambda}_{n}, \beta ; \frac{k a^{2+\theta}}{(2+\theta) \mathcal{D}}\right) \Psi\left(\bar{\lambda}_{n}, \beta ; \frac{k b^{2+\theta}}{(2+\theta) \mathcal{D}}\right)=0
$$

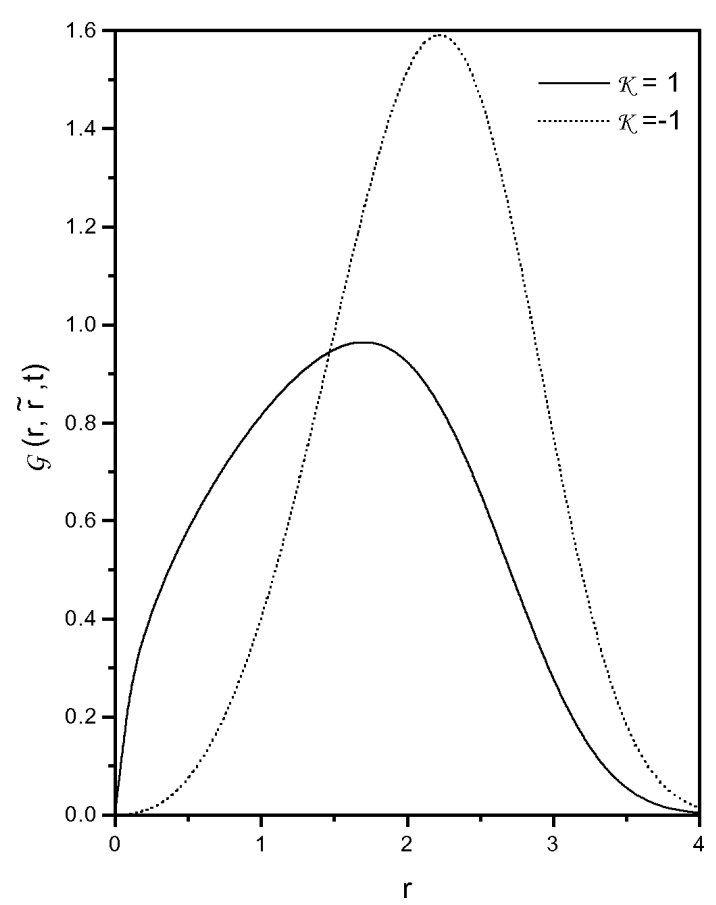

Fig. 4. Behaviour of $\mathcal{G}(r, \tilde{r}, t)$ versus $r$ which illustrates equation (23) for typical values of $\mathcal{K}$ by considering, for simplicity, $\mathcal{D}=1, \mathcal{N}=2, t=1, \tilde{r}=1$, and $\theta=1$. and the normalization condition is given by $\overline{\mathcal{N}}_{n}=$ $1 / \int_{a}^{b} \mathrm{~d} r r^{\mathcal{N}-1-\frac{\mathcal{K}}{\mathcal{D}}} \mathrm{e}^{-\frac{k r^{2+\theta}}{(2+\theta) \mathcal{D}}} \bar{\psi}_{n}^{2}\left(r, \lambda_{n}\right)$. Notice that $(24)$ may be extended to the interval $0 \leq r<\infty$ by taking the limit $a \rightarrow 0$ and $b \rightarrow \infty$ accomplishing the boundary condition $\lim _{b \rightarrow \infty} \rho(b, t)=0$. In this direction, after some calculations, it is possible to show that the solution is given by

$$
\begin{aligned}
& \rho(r, t)= \\
& -\frac{1}{\Gamma(1-\gamma)} \int_{0}^{t} \mathrm{~d} t \frac{1}{(t-\tilde{t})^{\gamma}} \int_{0}^{\infty} \mathrm{d} \tilde{r} \tilde{r}^{\mathcal{N}-1} \bar{\rho}(\tilde{r}) \mathcal{G}(r, \tilde{r} ; s) \\
& -\left.a^{\mathcal{N}-\theta-1} \int_{0}^{t} \mathrm{~d} \bar{t} \int_{0}^{\bar{t}} \mathrm{~d} t^{\prime} \mathcal{D}(t-\bar{t}) \phi_{a}\left(\bar{t}-t^{\prime}\right) \frac{\partial}{\partial r^{\prime}} \mathcal{G}\left(r, r^{\prime} ; t^{\prime}\right)\right|_{r^{\prime}=a}
\end{aligned}
$$

with the Green function

$$
\begin{aligned}
& \mathcal{G}\left(r, r^{\prime}, t\right)=-r^{\frac{\mathcal{K}}{\mathcal{D}}} t^{\gamma-1} \mathrm{e}^{-\frac{k r^{2+\theta}}{(2+\theta) \mathcal{D}}} \\
& \cdot \sum_{n=1}^{\infty} \widehat{\mathcal{N}}_{n} \hat{\psi}_{n}\left(r, \lambda_{n}\right) \hat{\psi}_{n}\left(r^{\prime}, \lambda_{n}\right) \mathrm{E}_{\gamma+\eta, \gamma}\left(-\mathcal{D} \lambda_{n}^{2} t^{\gamma+\eta}\right)
\end{aligned}
$$

with the eigenfunction

$$
\hat{\psi}_{n}\left(r, \lambda_{n}\right)=\mathrm{L}_{n}^{\xi}\left(\frac{k r^{2+\theta}}{(2+\theta) \mathcal{D}}\right)
$$


and

$$
\hat{N}_{n}=\left(\frac{k}{(2+\theta) \mathcal{D}}\right)^{\frac{\mathcal{K}+\mathcal{N} \mathcal{D}}{(2+\theta) \mathcal{D}}} \frac{(2+\theta) \Gamma(n+1)}{\Gamma\left(\frac{\mathcal{K}+\mathcal{N D}}{(2+\theta) \mathcal{D}}+n\right)}
$$

where $\xi=(\mathcal{K}+\mathcal{N D}) /[(2+\theta) \mathcal{D}]-1, \mathrm{~L}_{n}^{\xi}(x)$ are associated Laguerre polynomials, and $\lambda_{n}=(2+\theta) n k$. It is interesting to note that the solution for cases analyzed above may present a stationary solution depending on the choice of the time dependent functions $\phi_{a}(t)$ and $\phi_{b}(t)$. This feature indicates that the fractional time derivative and the form of the time dependence of the diffusion coefficient may be useful to describe systems with anomalous relaxation which have as stationary solution the usual one.

\section{Conclusion and Discussion}

We investigated a $\mathcal{N}$-dimensional fractional diffusion equation with radial symmetry accomplishing external forces and spatial and time dependent diffusion coefficient. We also consider the arbitrary reaction term $\alpha(r, t)$. Equation (1) is firstly analyzed by consid-

[1] J. Crank, The Mathematics of Diffusion, Oxford University Press, London 1956.

[2] P. Brault, C. Josserand, J. M. Bauchire, A. Caillard, C. Charles, and R. W. Boswell, Phys. Rev. Lett. 102, 045901 (2009).

[3] Y. Caspi, D. Zbaida, H. Cohen, and M. Elbaum, Macromolecules 42, 760 (2009).

[4] C. D. Ball, N. D. MacWilliam, J. K. Percus, and R. K. Bowles, J. Chem. Phys. 130, 054504 (2009).

[5] V. V. Saenko, Plasma Physics Reports 35, 1 (2009).

[6] J. Klafter and G. Zumofen, Phys. Rev. E 49, 4873 (1994).

[7] R. Metzler, G. Glöckle, and T. F. Nonnenmacher, Physica A 211, 13 (1994).

[8] A. Pekalski and K. Sznajd-Wero, Anomalous Diffusion: From Basics to Applications, Lecture Notes in Physics, Springer-Verlag, Telos 1999.

[9] M. F. Shlesinger, G. M. Zaslavsky, and U. Frisch, Lévy Flights and Related Topics in Physics, Lecture Notes in Physics, Springer-Verlag, Berlin 1994.

[10] B. J. West, M. Bologna, and P. Grigolini, Physics of Fractal Operators, Springer, New York 2002.

[11] T.D. Frank, Nonlinear Fokker-Planck Equations, Springer-Verlag, Heidelberg 2005.

[12] R. Hilfer, Applications of fractional calculus in physics, World Scientific, Singapore 2000. ering the spatial and time dependent diffusion coefficient $\mathcal{D}(r, t)=\overline{\mathcal{D}}(t) r^{-\theta}$, where $\overline{\mathcal{D}}(t)=\overline{\mathcal{D}} t^{\eta-1} / \Gamma(\eta-1)$, without external forces and reaction term subjected to a time dependent boundary conditions. The solution obtained for this case shows an anomalous behaviour which may be verified by analyzing the distribution or the mean square displacement. The survival probability also manifests a non-usual behaviour, in particular, when the boundary condition is given by $\rho(a, t)=$ $\rho(b, t)=0$ the asymptotic behaviour is $\mathcal{S}(t) \sim 1 / t^{\gamma+\eta}$. Afterwards, we have incorporated external forces in our analysis and have seen that they play an important role on the diffusion process by affecting the spreading of the distribution and consequently of the system. Finally, we expect that the results found here may be useful to investigate situations where the fractional diffusion equations are present.

\section{Acknowledgement}

We thank CNPq/INCT-SC, CAPES, and Fundação Araucária (Brazilian Agencies) for the financial support.

[13] R. Hilfer, R. Metzler, A. Blumen, and J. Klafter (Eds.), Strange Kinetics, Chemical Physics 284, PergamonElsevier, Amsterdam 2004.

[14] R. Metzler and J. Klafter, Phys. Rep. 339, 1 (2000).

[15] G. M. Zaslavsky, Phys. Rep. 371, 4612002.

[16] W. T. Coffey, D. S. F. Crothers, D. Holland, and S. V. Titov, J. Mol. Liq. 114, 165 (2004).

[17] S. Eule, R. Friedrich, F. Jenko, and D. Kleinhans, J. Phys. Chem. B 111, 11474 (2007).

[18] R. Metzler, E. Barkai, and J. Klafter, EuroPhys. Lett. 46, 431 (1999).

[19] P. C. Assis, P. C. da Silva, L. R. da Silva, E. K. Lenzi, and M. K. Lenzi, J. Math. Phys. 47, 103302 (2006).

[20] G. Gonçalves, M. K. Lenzi, L. S. Moraes, E. K. Lenzi, and M. F. Andrade, Acta Scientiarum Technology 27, 123 (2005).

[21] F. Y. Ren, J. R. Liang, W. Y. Qiu, X. T. Wang, Y. Xu, and R. Nigmatullin, Phys. Lett. A 321, 187 (2003).

[22] S. A. El-Wakil, A. Elhanbaly, and M. A. Zahran, Chaos, Solutions, and Fractals 12, 1035 (2001).

[23] B. I. Henry, T. A. M. Langlands, and S.L. Wearne, Phys. Rev. E 74, 031116 (2006).

[24] K. Seki, M. Wojcik, and M. Tachiya, J. Chem. Phys. 119, 2165 (2003).

[25] S. B. Yuste, L. Acedo, and K. Lindenberg, Phys. Rev. E 69, 036126 (2004). 
[26] I. M. Sokolov, M. G. W. Schmidt, and F. Sagués, Phys. Rev. E 73, 031102 (2006).

[27] Y.Z. Povstenko, J. Mol. Liq. 137, 46 (2008).

[28] M. A. Zahran, Z. Naturforsch. 63a, 553 (2008).

[29] B. N. N. Achar and J. W. Hanneken, J. Mol. Liq. 114, 147 (2004).

[30] T. Bickel, Physica A 377, 24 (2007).

[31] R. Rossato, M. K. Lenzi, L. R. Evangelista, and E. K. Lenzi, Phys. Rev. E 76, 032102 (2007).

[32] L. S. Lucena, L. R. da Silva, L. R. Evangelista, M. K. Lenzi, R. Rossato, and E. K. Lenzi, Chem. Phys. 344, 90 (2008).

[33] I. Podlubny, Fractional Differential Equations, Academic Press, San Diego 1999.

[34] M. P. Morse and H. Feshbach, Methods of Theoretical Physics, McGrawHill, New York 1953.

[35] R. S. Zola, E. K. Lenzi, L. R. Evangelista, and G. Barbero, Phys. Rev. E 75, 0422601 (2007).

[36] M. A. Lomholt, I. M. Zaid, and R. Metzler, Phys Rev. Lett. 98, 200603 (2007).
[37] S. Condamin, O. Bénichou, and J. Klafter, Phys. Rev. Lett. 98, 250602 (2007).

[38] E. K. Lenzi, R. S. Mendes, J. S. Andrade Jr., L. R. da Silva, and L.S. Lucena, Phys. Rev. E 71, 052101 (2005).

[39] I. M. Sokolov, J. Klafter, and A. Blumen, Phys. Rev. E 61, 2717 (2000).

[40] A. A. Vedenov, Rev. Plasma Phys. 3, 229 (1967).

[41] S. Redner, A Guide to First-Passage Processes, Cambridge University Press, Cambridge 2001.

[42] P. Macheras and A. Iliadis, Modeling in Biopharmaceutics, Pharmacokinetics, and Pharmacodynamics, Springer, New York 2006.

[43] A. Iomin, Phys. Rev. E 73, 061918 (2006).

[44] H.S. Carslaw and J. C. Jaeger, Conduction of Heat in Solids, Oxford University Press, London 1959.

[45] E. K. Lenzi, L. R. da Silva, A. T. Silva, L. R. Evangelista, and M. K. Lenzi, Physica A 388, 806 (2009).

[46] A. M. Mathai and R. K. Saxena, The H-function with Application in Statistics and other Disciplines, Wiley Eastern, New Delhi 1978. 